\title{
Assessment of minimally invasive direct coronary artery bypass grafting of the left internal thoracic artery by means of magnetic resonance imaging
}

\author{
Norbert I. Stauder, MD \\ Michael Fenchel, MD \\ Heidrun Stauder, $M D^{\mathrm{b}}$ \\ Axel Küttner, $\mathrm{MD}^{\mathrm{a}}$ \\ Albertus M. Scheule, MD \\ Ulrich Kramer, MD ${ }^{\mathrm{a}}$ \\ Claus D. Claussen, $\mathrm{MD}^{\mathrm{a}}$ \\ Stephan Miller, MD ${ }^{a}$
}

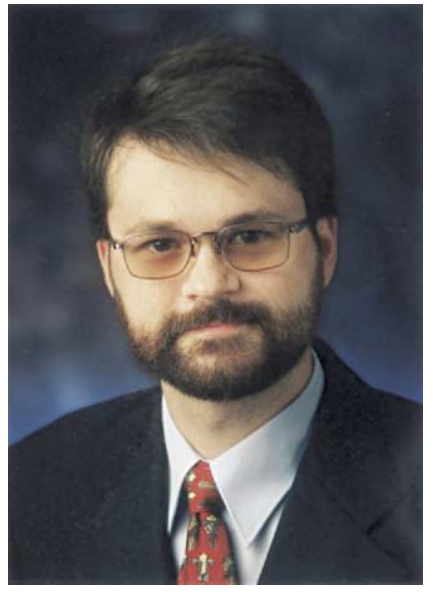

Dr Norbert I. Stauder

\footnotetext{
From the Department of Diagnostic Radiology $^{\mathrm{a}}$ and the Department of Thoracic, Cardiac and Vascular Surgery, ${ }^{\mathrm{b}}$ EberhardKarls-University, Tuebingen, Germany.

Received for publication April 30, 2004; revisions received June 28, 2004; accepted for publication July 8, 2004.

Address for reprints: Norbert I. Stauder, MD, Department of Diagnostic Radiology, Eberhard-Karls-University, Tuebingen, Hoppe-Seyler-Str. 3, Germany, 72076 Tuebingen (E-mail: norbert.stauder@med. uni-tuebingen.de)

J Thorac Cardiovasc Surg 2005;129:607-14 $0022-5223 / \$ 30.00$

Copyright (C) 2005 by The American Association for Thoracic Surgery

doi:10.1016/j.jtcvs.2004.07.064
}

Objectives: We sought to evaluate graft patency, flow, and flow reserve in patients with minimally invasive direct coronary artery bypass surgery of internal thoracic artery grafts by a combined magnetic resonance protocol with a phase-contrast technique and magnetic resonance angiography.

Methods: At 1.5 T (Magnetom Sonata, Siemens), 30 symptomatic patients with 30 left internal thoracic artery grafts were examined 6 years after minimally invasive surgical intervention. Navigator-gated magnetic resonance angiography and contrast-enhanced FLASH-3D magnetic resonance angiography $(0.2 \mathrm{mmol}$ gadopentate-diethylene triamine pentetic acid [Gd-DTPA] $/ \mathrm{kg}$ body weight) was used to assess bypass patency. Phase-contrast flow measurements with retrospective gating were performed in the internal thoracic artery grafts at rest and after stress induction with dipyridamole $(0.57$ $\mathrm{mg} / \mathrm{kg}$ body weight). Graft patency was evaluated by means of multidetector computed tomography (Sensation 16, Siemens).

Results: Internal thoracic artery grafts were occluded in 5 of 30 patients. In 6 patients the anastomosis to the left anterior descending artery was highly stenotic $(>70 \%)$ at multidetector computed tomography. In patients with regular grafts (multidetector computed tomography), a significant improvement of graft flow ( $P<$ $.001)$ and diastolic/systolic peak velocity ratio $(P<.001)$ after stress induction was detected. Magnetic resonance angiography combined with flow reserve measurements could differentiate between occluded-stenotic and regular minimally invasive direct coronary artery bypass grafts.

Conclusions: Magnetic resonance imaging allows a combined assessment of bypass patency and flow with flow reserve in patients after the minimally invasive direct coronary artery bypass operation. The protocol of this study might be applicable for the evaluation of graft status in symptomatic patients after revascularization.

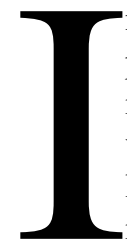

n patients with coronary artery disease, surgical myocardial revascularization is performed to improve myocardial function. Treatment is either by means of internal thoracic artery (ITA) coronary artery bypass grafting or homologous venous coronary artery bypass grafting. In patients with isolated stenoses of the left anterior descending artery (LAD) or the right coronary artery, minimally invasive direct coronary artery bypass grafting (MIDCAB) of the ITA through a small left anterior thoracotomy without cardiopulmonary bypass has been used with 
increasing frequency in the past decade. ${ }^{1}$ However, thoracic discomfort can persist in a subgroup of patients after surgical therapy. Previous studies revealed an incidence of bypass occlusion or stenosis during the first year of up to $15 \%$ for MIDCAB grafts. ${ }^{2}$ At present, the diagnostic method of choice in this situation is invasive catheter angiography to investigate coronary artery and bypass status. However, catheter angiography is an invasive procedure with a risk of minor and major complications and necessitates radiographic exposure. ${ }^{3}$ Today, multidetector computed tomography (MDCT) allows accurate assessment of patency in bypass grafts. ${ }^{4,5}$ Magnetic resonance imaging (MRI), on the other hand, provides information about graft morphology and function. ${ }^{6-11}$ Although the morphologic delineation of the bypass grafts could be improved by means of contrast-enhanced 3-dimensional magnetic resonance (3-D MR) angiography (ceMRA), visualization of the vascular mediastinal course was still hampered by clip artifacts, and the depiction of the distal anastomosis remained problematic.,7 Recently introduced, high-resolution, navigator-gated 3-D MRA is a promising technique that allows the differentiation of patent and occluded grafts and also allows the assessment of vein graft disease with a sufficient diagnostic accuracy. ${ }^{8}$ Furthermore, it was found that information about the function of conventional internal thoracic bypass grafts could be obtained by means of quantitative flow and flow reserve measurements. ${ }^{9,10}$ A combination of flow reserve measurements, ceMRA, and navigator-based MRA for distal anastomoses might be beneficial in improving the noninvasive diagnostic evaluation of MIDCAB grafts by means of MRI.

The purpose of this study was to evaluate an MR protocol for morphologic and functional assessment of MIDCAB grafts by using different MRA techniques and flow measurements at rest and after medicamentous stress induction.

\section{Patients and Methods \\ Patient Study}

A total of 70 patients underwent MIDCAB at our hospital from 1997 through 1999. The MIDCAB technique used a minithoracotomy in the fourth or fifth left anterior intercostal space. The distal left ITA (LITA) was skeletonized between the third and sixth intercostal spaces. The end-to-side anastomosis to the LAD was performed with a mechanical stabilizer (CardioThoracic Systems Inc) and consecutive sutures of 7-0 or 8-0 Prolene (Ethicon, Inc, Somerville, NJ). Thirty consecutive symptomatic (ie, atypical chest pain, angina, or intermittent thoracic discomfort) patients (24 male and 6 female patients; mean age, $65.3 \pm 8.4$ years) with 30 LITA grafts were examined $6.1 \pm 0.75$ years (range, 60-88 months) after MIDCAB surgery. The MIDCAB grafts were exclusively solitary grafts anastomosed to the LAD. Medical history was obtained from patients postoperatively concerning chest discomfort and amount of tolerable exercise. Participation in the study was voluntary, and patients were enrolled after providing written informed consent. The study was approved by the ethics committee of our hospital. All patients were checked by a cardiologist and a heart surgeon.

\section{MR Imaging}

MR examinations were performed at $1.5 \mathrm{~T}$ (MAGNETOM Sonata Maestro Class, Siemens Medical Solutions) by using a 6-channel phased-array surface coil and electrocardiographic (ECG) triggering. At first, an axial HASTE sequence (repetition time [TR] $800 \mathrm{~ms}$ adapted to RR interval; echo time [TE], $26 \mathrm{~ms}$; flip, $16^{\circ}$; field of view [FOV], $360 \times 360 \mathrm{~mm}^{2}$; matrix, $256 \times 256 \mathrm{~mm}^{2}$; slice thickness, $5 \mathrm{~mm}$ ) was applied for the examination of mediastinal anatomy and location of the bypass graft. For the assessment of the distal part of the MIDCAB graft and the distal anastomosis to the LAD, an ECGtriggered, navigator-gated 3-D MRA scan (TR, $160 \mathrm{~ms}$; TE, $2.05 \mathrm{~ms}$; flip, $25^{\circ}$; voxel, $0.9 \times 0.6 \times 1.2 \mathrm{~mm}^{3}$; TD approximate, $500 \mathrm{~ms}$ adapted to the RR interval; slices per slab, 26) was applied. Data were acquired in mid-diastole with an acquisition window of $180 \mathrm{~ms}$ by using a heart rate-dependent trigger delay. The determination of the bolus transit time was done with a Turbo FLASH 2-dimensional sequence. A power injector (Medrad Spectris, Volkach) was used to inject $3 \mathrm{~mL}$ of gadopentate-diethylene triamine pentetic acid (GdDTPA; Magnevist, Schering) for the evaluation of transit time and 0.2 mmol Gd-DTPA (Magnevist)/kg body weight for the ceMRA, respectively, both followed by $30 \mathrm{~mL}$ of $0.9 \% \mathrm{NaCl}$ into an antecubital vein at a flow rate of $2.5 \mathrm{~mL} / \mathrm{s}$. For the ceMRA, a 3-D FLASH sequence with the following parameters was used: TR, $2.5 \mathrm{~ms}$; TE, 1 $\mathrm{ms}$; flip angle, $20^{\circ}$; FOV, $300 \times 300 \mathrm{~mm}^{2} ; 1.4 \times 0.9 \times 1.0 \mathrm{~mm}^{3}$ fat saturation. After the course of the MIDCAB graft was localized, flow measurements were performed at the level of the bifurcation of the pulmonary trunk perpendicular to the course of the vessel. After resting flow measurements, $0.57 \mathrm{mg}$ of dipyridamole per kilogram of body weight was injected over 4 minutes. Flow measurements were repeated 2 minutes after finishing the dipyridamole injection. Heart rate and blood pressure were monitored during the entire examination. For the flow measurements, a segmented phase-contrast FLASH 2-dimensional sequence (TReff, $25 \mathrm{~ms}$; TE, $3.2 \mathrm{~ms}$; flip angle, $30^{\circ}$; FOV, $280 \times 280 \mathrm{~mm}$; matrix, $256 \times 256 \mathrm{~mm}^{2}$; slice thickness, 5 $\mathrm{mm}^{2}$; velocity encoding, $90 \mathrm{~cm} / \mathrm{s}$; spatial resolution, $1.1 \times 1.1 \times 5$ $\mathrm{mm}^{3}$; retrospective gating) was used.

\section{MR Image Analysis}

Two experienced investigators blinded to the results of MDCT evaluated all ceMR angiograms and navigator-gated MR angiograms. Image evaluation was performed on a Leonardo workstation (Siemens Medical Systems) by analyzing the source images of the 3-D data sets, as well as multiplanar reconstruction and maximum intensity projection in a consensus reading. For data analysis, the LITA graft was subdivided into 5 segments: branching from the subclavian artery and the proximal third, middle segment, distal third, and anastomosis to the LAD. In ceMRA images and navigator-gated MRA images, a MIDCAB graft was considered patent if the full course of the vessel, including the distal anastomosis, was visible. If there was an interruption of the vessel course or a $70 \%$ or greater luminal diameter narrowing in the graft course, the vessel was rated stenotic $(\geq 70 \%)$.

The subjective quality of bypass delineation was ranked on a 4-point scale: 3, excellent; 2 , diagnostic; 1 , poor; and 0 , not visible.

For image postprocessing and flow quantification, a semiautomated software program (ARGUS, Siemens) was used. The lumina of the grafted ITAs were identified on magnitude images of the flow quantification sequence. A region of interest was drawn 
TABLE 1. Delineation scores* of minimally invasive direct coronary artery bypass grafts by means of contrast-enhanced 3-dimensional magnetic resonance angiography

\begin{tabular}{lccccc}
\hline MIDCAB & Begin & Proximal part & Middle part & Distal part & Anastomosis \\
\hline Group A & $2.6 \pm 0.6$ & $2.8 \pm 0.4$ & $2.9 \pm 0.3$ & $2.5 \pm 0.8$ & $1.9 \pm 1.3$ \\
Group B & $2.2 \pm 0.8$ & $2.6 \pm 0.9$ & $2.1 \pm 1.2$ & $0.8 \pm 0.8$ & $0.6 \pm 1$ \\
Significance & $P=\mathrm{NS}$ & $P=\mathrm{NS}$ & $P<.02$ & $P<.0001$ & $P<.02$ \\
Group B' & $2.2 \pm 0.8$ & $2.9 \pm 0.4$ & $2.4 \pm 0.9$ & $1.4 \pm 0.5$ & $1.4 \pm 0.9$ \\
Significance & $P=\mathrm{NS}$ & $P=\mathrm{NS}$ & $P<.05$ & $P<.01$ & $P<.02$ \\
\hline
\end{tabular}

In group $\mathrm{B}^{\prime}$ the occluded grafts are excluded. Patent grafts could be significantly better depicted in the middle and distal part as stenotic grafts. MIDCAB, Minimally invasive direct coronary artery bypass; $L A D$, left anterior descending artery; $N S$, Not significant.

$*$ Score $0-3: 0=$ not visible to $3=$ excellently visible.

manually around the lumen. A reference region of interest was placed into adjacent mediastinal fat to correct flow measurements far craniocaudal respiratory motion. The MIDCAB graft mean flow values at baseline, as well as after stress induction and mean flow reserve, were determined as absolute values in milliliters per minute. Furthermore, index values corrected for body surface area, the diastolic/systolic peak velocity ratio, and the flow reserve ratio were calculated.

\section{MDCT}

MDCT data sets were acquired on average within 3 hours (2-24 hours) of the MR examination by using a 16-detector CT scanner (Sensation 16, Siemens Medical Systems). $\beta$-Receptor blocking medication (100 mg of metroprolol) was used before scanning to reduce the heart rate if it was higher than 75 beats $/ \mathrm{min}$. To evaluate the circulation time, $20 \mathrm{~mL}$ of contrast media $(20 \mathrm{~mL}$ at $4 \mathrm{~mL} / \mathrm{s}$, $400 \mathrm{mg}$ of iodine/mL; Imeron 400, Altana Pharma) and a chaser bolus of $20 \mathrm{~mL}$ of saline was administrated in an antecubital vein. The correct scanning delay was established by measuring CT attenuation values in the ascending aorta by using the first slice after maximum contrast as circulation time. By using a dual-head and power injector (CT2TM, Medtron Saarbrücken), a total volume of $100 \mathrm{~mL}$ of intravenous contrast agent plus $20 \mathrm{~mL}$ of chaser bolus was injected $(50 \mathrm{~mL}$ at $4 \mathrm{~mL} / \mathrm{s}$ and then $50 \mathrm{~mL}$ at $2.5 \mathrm{~mL} / \mathrm{s})$. A contrast-enhanced scan was acquired by using the following protocol: $0.75-\mathrm{mm}$ collimation; table feed, $3.8 \mathrm{~mm} /$ rotation; tube current, 500 effective $\mathrm{mA}$ at $140 \mathrm{kV}$.

For image reconstruction, the standard built-in reconstruction algorithm was used. The reconstruction window was set to start at a $60 \%$ RR interval for all native images, as well as for the contrast-enhanced scan, to determine the reconstruction interval with the fewest motion artifacts. If necessary, a test series reconstructing slices ranging from $35 \%$ to $75 \%$ relative to the RR interval was performed in $2 \%$ steps. The time point with the least motion artifacts was chosen to reconstruct the entire stack of images of the CT angiography scan. Depending on the individual case, axial slices and advanced postprocessing tools, such as multiplanar reconstruction and maximum intensity projection, were used. Patients with chest pain were referred to invasive angiography when graft stenosis-occlusion or a relevant coronary stenosis elsewhere was diagnosed by means of MDCT. Total scan and reconstruction time was, on average, 35 minutes (range, 25-45 minutes).

\section{Statistical Analysis}

All values were expressed as mean values and SDs. The significance of differences between 2 groups (patients without and with significant stenosis) was determined by using a 2-tailed $t$ test. The statistical significance between MR flow measurements at baseline and after stress induction was determined by using a paired 2 -tailed $t$ test. Interobserver and intraobserver variability of flow measurements were determined by 2 independent investigators using the Bland Altman approach.

\section{Results}

MDCT

In 19 patients the MIDCAB graft was patent without significant graft stenosis. In 11 patients a significant graft stenosis ( $\geq 70 \%$ luminal diameter narrowing) was found at the distal anastomosis to the LAD or in the distal part of the graft, with 5 grafts being completely occluded in the region where the LITA was detached from the thoracic wall. The 30 patients were assigned to 2 groups according to the assessment of stenosis at MDCT: group A comprised 19 patients without significant stenosis, and group B comprised 11 patients with significant graft stenosis, including the 5 patients with occluded grafts (100\% stenosis).

MR examinations could be performed in all patients according to the protocol. Three (10\%) patients reported mild headache. The heart rate increased significantly after dipyridamole administration from $62.8 \pm 8.3$ beats $/ \mathrm{min}$ at rest to $79.4 \pm 8.4$ beats/min after stress induction $(P<$ $.001)$.

\section{MR Angiography}

By ceMRA, an adequate image quality could be obtained in all patients. Navigator-gated MRA could be estimated in 29 patients, whereas 5 examinations could not be evaluated as a result of poor image quality. Examination time of navigatorbased angiography was $16.3 \pm 5.1$ minutes.

All 5 occluded grafts could be evaluated with ceMRA. In 17 patients a definite determination about graft patency was possible because the distal anastomosis to the LAD could be evaluated. Eleven of those distal anastomoses were excel- 


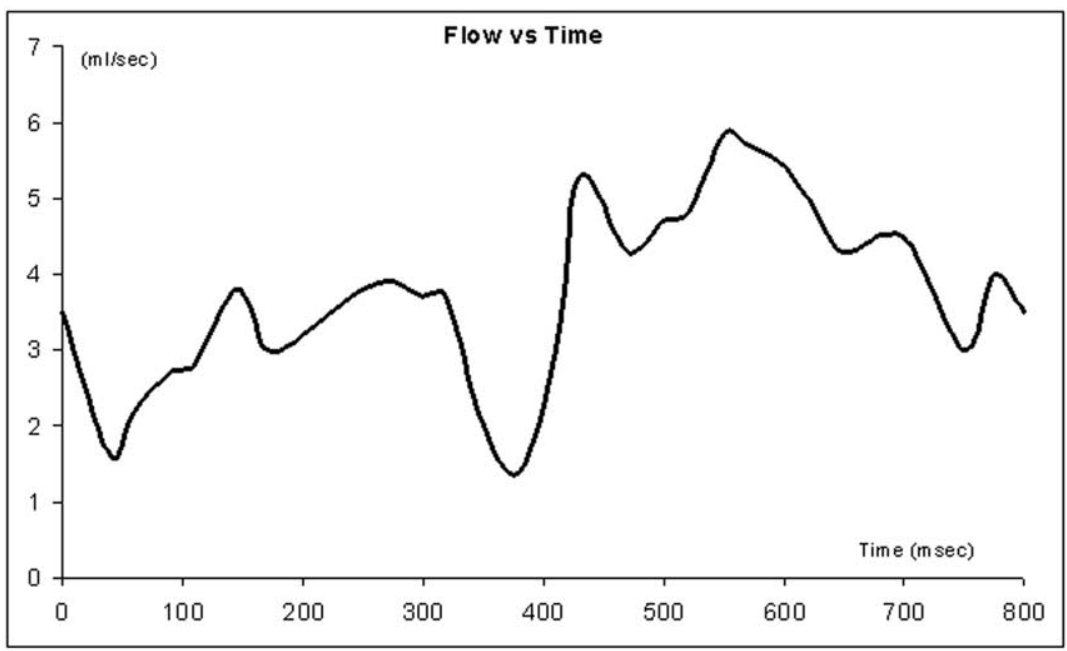

Figure 1. Representative flow quantification in a patent MIDCAB graft with a biphasic flow pattern with predominant diastolic peak.

lently depicted, with a subjective image quality score of 3 . Delineation scores of the 2 groups are summarized in Table 1. On ceMRA, 7 anastomoses to the LAD could not be definitely evaluated, and therefore those grafts were considered stenotic. Sensitivity and specificity for identifying graft stenosis were $100 \%$ and $68 \%$, respectively. With the additional evaluation of navigator-gated MRA images from 7 ambiguous anastomoses, 5 could be evaluated, and therefore only 2 grafts could not be morphologically classified. Those grafts were considered stenotic. The specificity increased to $89 \%$.

\section{Flow Measurements}

Adequate flow measurements at baseline and after stress induction were acquired in all patients. In patients with suspected occlusion of the bypass, as determined with ceMRA, the flow measurements were performed proximal to the occluded region. Intraobserver and interobserver variability of flow measurements were $9 \%$ and $16 \%$ at baseline and $7 \%$ and $14 \%$ after stress induction, respectively.

The typical flow pattern of the native ITA vessels shows one systolic maximum. In contrast, the characteristic flow pattern in a patent LITA graft is biphasic, with a lower systolic and a higher diastolic peak. In group A (the patients without significant stenosis) the flow pattern was similarly characterized by a predominant flow during the diastolic phase (Figure 1). In contrast, diastolic peak flow was reduced and systolic flow was predominant in group B (patients with significant graft stenosis or occlusion, Figure 2). Diastolic/systolic peak velocity ratios at baseline and after stress induction, flow values at baseline and after application of dipyridamole, and flow reserve data are summarized in Table 2.

\section{Combination of Flow With ceMRA and Navigator- based MRA}

Total examination time was $49.4 \pm 8.9$ minutes. By means of combined evaluation of all MR methods (ie, flow measurements at baseline and stress, ceMRA, and navigatorbased MRA), all severe graft stenoses (5 grafts) and occluded grafts (5 grafts) could be identified in comparison with MDCT. All patent grafts without stenosis could be detected. Sensitivity, specificity, and positive and negative predictive values of all MR methods are summarized in Table 3.

\section{Further Management of Patients With Stenotic and Occluded Grafts}

All patients were checked by a cardiologist and a heart surgeon. In 19 patients without pathologic image findings, scar disorders were attributable to their mild and partially intermittent or meteorosensitive atypical chest discomfort. In 11 patients with angina and stenotic or occluded grafts at MDCT and in 1 patient with relevant stenosis at MDCT in the right coronary artery, coronary angiography was performed. Coronary angiography confirmed the results of MDCT and MRI in all patients. In 3 patients with occluded grafts, re-revascularization was performed by means of venous aorta-coronary bypass grafting to the LAD or ramus diagonalis. In 2 patients with large infarcted areas and reduced cardiac output presenting with severe coronary artery disease, no further surgical therapy was performed. In one patient with a stenotic graft, successful percutaneous coronary angioplasty was performed in the proximal LAD. In 1 patient with a stenotic distal anastomosis, the distal LAD was occluded at MDCT, and a circumscribed infarcted area with thin scar tissue was found on cine images. No 


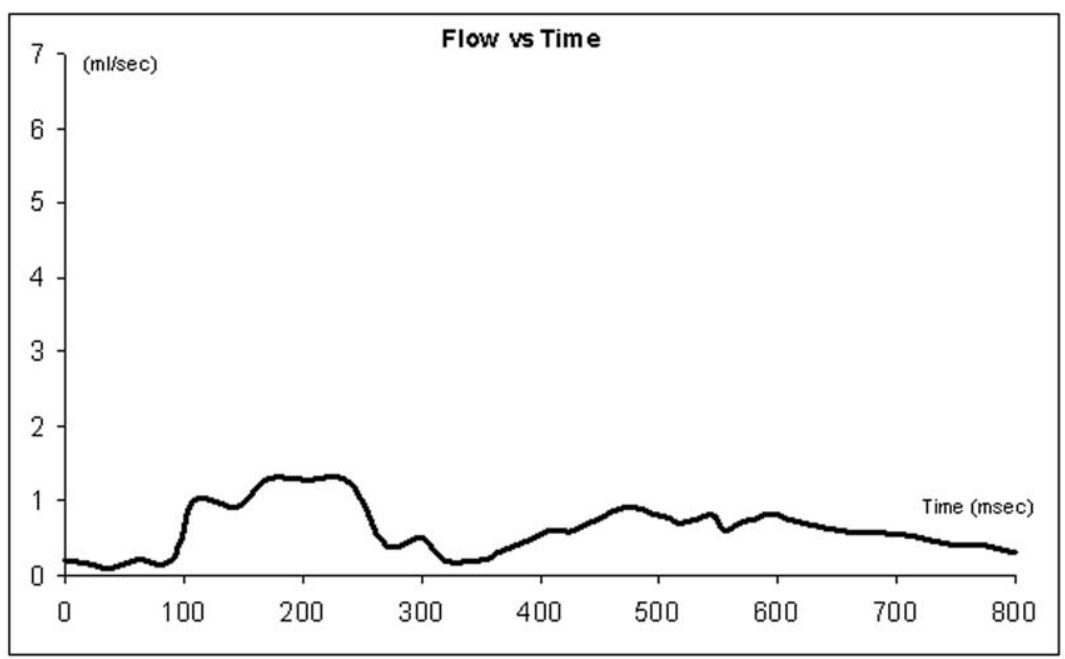

Figure 2. Representative flow quantification in a stenotic MIDCAB graft with a biphasic flow pattern with predominant systolic peak.

further revascularization therapy could be performed. In 4 patients with graft stenosis, reduced mean flow and flow reserve, and mild angina but sufficient myocardial function (ejection fraction $>50 \%$ ), a conservative therapy management with continuous monitoring was started. In 1 patient with a stenosis in the proximal right coronary artery, percutaneous coronary angioplasty was performed.

\section{Discussion}

In the present study MRI was used for noninvasive follow-up of patients after MIDCAB surgery. The imaging protocol comprised several important properties of the MIDCAB grafts, such as bypass patency, as well as the flow at baseline and after pharmacologic stress induction. As a key finding, we observed significant differences in baseline blood flow between regular and stenotic grafts. All occluded grafts could be detected in ceMRA images. The diastolic/ peak velocity ratio and flow reserve ratio are not suitable as parameters for detecting graft stenosis in patients with MIDCAB grafts. The reduced delineation of the distal anastomosis to the LAD in ceMRA images could be improved by navigator-gated MRA.

\section{MR Angiography}

Since the beginning of the 1990s, several methods have been investigated for the assessment of coronary bypass grafts by MRI. With the introduction of ceMRA, sensitivities for bypass patency between $93 \%$ and $95 \%$ and specificities between $67 \%$ and $97 \%$ were found. ${ }^{6,12,13}$

Several studies reported difficulties in the evaluation of ITA grafts because of the small-vessel diameter and surgical clip artifacts. ${ }^{14,15}$ Such artifacts in the region of the LITA also proved to be a limiting factor in some cases of our
TABLE 2. Values of diastolic/systolic peak velocity ratios, mean flow at baseline, and, after stress induction, flow reserve and flow reserve ratio in patients after minimally invasive direct coronary artery bypass grafting surgery

\begin{tabular}{lccc}
\hline MIDCAB & Group A & Group B & Significance \\
\hline D/S PVR base & $1.1 \pm 0.35$ & $0.9 \pm 0.58$ & NS \\
D/S PVR stress & $1.5 \pm 0.3$ & $0.9 \pm 0.5$ & $P<.001$ \\
Flow base $(\mathrm{mL} / \mathrm{min})$ & $79.5 \pm 48.3$ & $19.0 \pm 15.2$ & $P<.001$ \\
Flow stress $(\mathrm{mL} / \mathrm{min})$ & $183.7 \pm 71.5$ & $37.0 \pm 26.7$ & $P<.001$ \\
Flow reserve $(\mathrm{mL} / \mathrm{min})$ & $105.3 \pm 52.7$ & $18.0 \pm 17.5$ & $P<.001$ \\
Flow reserve ratio & $2.85 \pm 1.17$ & $2.04 \pm 0.96$ & $\mathrm{NS}$ \\
\hline
\end{tabular}

Patent grafts were included in group $A$, and grafts with significant stenosis $(\geq 70 \%$ luminal diameter narrowing at MDCT) were included in group $B$. $M I D C A B$, Minimally invasive direct coronary artery bypass grafting; $D / S$ PVR, diastolic/systolic peak velocity ratio; NS, Not significant.

study, especially when maximum-intensity projection reconstructions were evaluated. In patent vessels the proximal and middle segments of the MIDCAB grafts were clearly visible (Table 1). The distal anastomosis to the LAD could be detected in $70 \%$ of all cases without occlusion, whereas in previous studies this region could not be assessed. ${ }^{6,16}$

Additionally, the flow measurements were planned on ceMRA images. New imaging techniques, such as parallel imaging in combination with ECG triggering, will probably have a great potential to improve the assessment of bypass segments adjacent to the heart because of the reduction of pulsation artifacts and increasing spatial resolution within an acceptable scan time.

Another alternative for the morphologic examination of the bypass grafts by means of MRI is the use of navigator techniques. ${ }^{8}$ Thus far, no clinical results are reported applying this 
TABLE 3. Sensitivity, specificity, positive predictive value, and negative predictive value of different magnetic resonance techniques and the combination of all magnetic resonance methods for detecting stenosis in minimally invasive direct coronary artery bypass grafts in comparison with multidetector computed tomography

\begin{tabular}{lcccc}
\hline MRI techniques & $\begin{array}{c}\text { Sensitivity } \\
(\%)\end{array}$ & $\begin{array}{c}\text { Specificity } \\
(\%)\end{array}$ & $\begin{array}{c}\text { Positive predictive } \\
\text { value }\end{array}$ & $\begin{array}{c}\text { Negative predictive } \\
\text { value }\end{array}$ \\
\hline ceMRA & 100 & 68 & 0.63 & 1.0 \\
ceMRA + n-MRA & 100 & 89 & 0.83 & 1.0 \\
Flow at baseline & 90 & 95 & 0.9 & 0.95 \\
Flow after stress & 90 & 100 & 1.0 & 0.95 \\
Flow reserve & 100 & 95 & 0.91 & 1.0 \\
Flow reserve ratio & 50 & 53 & 0.36 & 0.67 \\
D/S PVR at baseline & 60 & 58 & 0.43 & 0.73 \\
D/S PVR after stress & 70 & 89 & 0.78 & 0.85 \\
Combination of all MR methods & 100 & 100 & 1.0 & 1.0 \\
\hline
\end{tabular}

MRI, Magnetic resonance imaging; ceMRA, contrast-enhanced 3-dimensional magnetic resonance angiography; $n$-MRA, navigator-gated magnetic resonance angiography; $D / S P V R$, diastolic/systolic peak velocity ratio; $M R$, magnetic resonance.

technique to MIDCAB grafts. Because of poor image quality, only $82 \%$ of the navigator-gated MRA examinations were evaluable in the present study, which corresponds to results obtained by Langerak and coworkers, ${ }^{8}$ who reported a success rate of $89 \%$ using navigator-gated MRA scans in vein grafts. However, the accuracy of detection of MIDCAB graft stenosis by means of ceMRA combined with navigator-gated MRA was considerably improved, from $79 \%$ to $93 \%$. As a limiting factor, the examination time was markedly longer (16 minutes, $33 \%$ of total examination time) by using navigator-gated MRA. Therefore, from a practical point of view, the application of navigator-based MRA is adequate for evaluating patients with MIDCAB grafts but not in patients with multiple vein grafts.

\section{Flow Measurements}

Flow measurements were found to be accurate in quantifying blood flow in coronary artery bypass grafts ${ }^{7-11,17}$ in addition to the morphologic delineation of bypass grafts. The blood flow pattern in regular MIDCAB grafts was found to be similar compared with that of conventional LITA grafts, ${ }^{7,10,17}$ with a diastolic/systolic peak velocity ratio of higher than 1.0 at baseline. However, the sensitivity $(60 \%)$ and specificity $(58 \%)$ of the diastolic/systolic peak velocity ratio at baseline for detecting stenosis were lower than in other studies. ${ }^{10,18} \mathrm{~A}$ significant difference of the diastolic/systolic peak velocity ratio between stenotic and nonstenotic MIDCAB grafts could be detected only after pharmacologic stress induction with a sensitivity and specificity of $70 \%$ and $89 \%$, respectively. In this regard our results differ from those of Ishida and associates, ${ }^{10}$ who reported that a diastolic/systolic peak velocity ratio less than 1.0 at baseline predicted significant LITA graft stenosis with a sensitivity and specificity of $86 \%$ and $88 \%$, respectively. This might be due to the minimally invasive operative technique, where the MIDCAB graft is only prepared a short distance of the LITA from the thoracic wall. It should be noted that systolic and diastolic blood flow patterns, as well as diastolic/systolic peak velocity ratios, in internal thoracic artery grafts and the optimal cutoff values can be substantially different, depending on the location of blood flow measurement. ${ }^{19}$ In contrast to patients operated on with the conventional technique, diastolic/systolic peak velocity ratios measured by means of MRI do not seem to be an effective diagnostic parameter for detecting graft stenosis in patients with MIDCAB grafts.

The highly reproducible baseline flow value in MIDCAB grafts with and without significant stenosis was concordant with previously reported results. ${ }^{10,20,21}$ The blood flow volume measurement is less influenced by the location of the measurement. Therefore this approach is advantageous in assessment of graft function after coronary bypass surgery. Patients with serious accompanying diseases that contraindicate pharmacologic stress induction could benefit from the evaluation of mean baseline blood flow. The evaluation of mean blood flow after stress induction and mean blood flow reserve led to an improvement of sensitivity and specificity for detection of graft stenosis (Table 3) and turned out to be an accurate diagnostic parameter for graft stenosis in MIDCAB grafts. In accordance with the results of Ishida and associates, ${ }^{10}$ in the flow reserve ratios between MIDCAB grafts with and without stenosis, no significant differences were observed in our study. A clinical application of flow measurements could be the serial assessment of graft function, which is useful in detecting gradual increases in graft stenosis before the onset of total occlusion.

\section{Combination Flow With ceMRA and Navigator-based MRA}

In the present study the combination of MRA techniques and flow quantification was beneficial for clinical practice. It is possible to compensate for the limitations of either method, and the diagnostic yield can be increased 
by using this approach. Clinically, the combined method could be useful in patients with severe contrast medium or iodine allergy or serious accompanying diseases, which contraindicate invasive diagnostic procedures. Also, there could be benefits in cases suspicious for occlusion of MIDCAB grafts or for patients with only a reduced number of coronary artery bypass grafts. The navigator-based MRA, in addition to flow measurements and ceMRA, is actually not practical in clinical practice in patients with multiple coronary bypass grafts because of the prolonged examination time. Future studies with ECG-triggered ceMRA scans with better spatial resolution or quicker navigator-based MRA scans in addition to flow measurements might allow a comprehensive evaluation of bypass grafts, including complete morphologic delineation and, in case of stenosis, its hemodynamic relevance. Nevertheless, a drawback of MRI remains the lack of simultaneous examination of the coronary arteries.

\section{Study Limitations}

A study limitation was the use of MDCT alone as a reference standard for graft patency in 19 patients. In the literature there are publications using the 4-detector CT for graft patency. The limitations mentioned for that scanner type are substantially reduced for the 16-detector CT used in this study. Nevertheless, some limitations and drawbacks of the CT technique in comparison with catheterization remained.

Generally, the CT scanning range is not extended to cover the entire course of the proximal LITA. However, in the patients undergoing MIDCAB in this study, the distal part of the LITA, used as MIDCAB, was covered in the scan volume in all patients. Extensive calcification of the coronary arteries and the degenerated grafts, as well as vascular clips in the proximity of arterial grafts, cause beam-hardening and partial volume artifacts that hamper the assessment of graft patency. These artifacts have been reduced since the introduction of 16-detector scanners with an increased rotation speed, but severe calcification (higher than an Agatston score of 1000) remains problematic and unfortunately remains a drawback of CT. Earlier disadvantages in examination of patients with arrhythmias and high heart rates decreased substantially with the introduction of new algorithms and the use of $\beta$-receptorblocking medication. The initially high radiation dose has been dramatically decreased since the introduction of ECG-triggered scan-modulation algorithms.

Despite these technical limitations, MDCT allows accurate assessment of graft patency, and in addition, it provides relevant information concerning the presence of substantial obstructive disease in the bypass grafts, including the distal anastomosis, and progression of disease of the coronary arteries. The technique is easy to perform and well tolerated and furthermore provides information about the coronary arteries.
Therefore MDCT can be used as a noninvasive examination for graft patency before catheterization in most of the patients.

MRI is feasible to assess graft morphology and flow reserve in patients after MIDCAB within an acceptable examination time. The approach renders noninvasive assessment of graft status after MIDCAB in patients with persistent chest pain. Therefore the method has a potential to help in identifying those patients who require further invasive diagnostic interventions after bypass grafting.

\section{References}

1. Calafiore AM, Di Giammarco G, Teodori G, Bosco G, D'Annunzio E, Barsotti A, et al. Left anterior descending coronary artery grafting via left anterior small thoracotomy without cardiopulmonary bypass. Ann Thorac Surg. 1996;61:1658-65.

2. Possati G, Gaudino M, Alessandrini F, Zimarino M, Glieca, F, Bruno P. Minithoracotomy myocardial revascularization: results of early angiographic control. G Ital Cardiol. 1997;27:749-57.

3. Johnson LW, Krone R. Cardiac catheterization 1991: a report of the Registry of the Society for Cardiac Angiography and Interventions (SCA\&I). Catheter Cardiovasc Diagn. 1993;28:219-20.

4. Silber S, Finsterer S, Krischke I, Lochow P, Muhling H. Noninvasive angiography of coronary bypass grafts with cardio-CT in a cardiology practice. Herz. 2003;28:126-35.

5. Nieman K, Pattynama PM, Rensing BJ, van Geuns R-JM, de Feyter PJ. Evaluation of patients after coronary artery bypass surgery: CT angiographic assessment of grafts and coronary arteries. Radiology. 2003;229: 749-56.

6. Wintersperger BJ, Engelmann MG, von-Smekal A, Knez A, Penzkofer HV, Hofling B, et al. Patency of coronary bypass grafts: assessment with breath-hold contrast-enhanced MR angiography: value of a nonelectrocardiographically triggered technique. Radiology. 1998;208: 345-51.

7. Miller S, Scheule AM, Hahn U, Jurmann M, Helber U, Duda SH, et al. MR angiography and flow quantification of the internal mammary artery graft after minimally invasive direct coronary artery bypass. Am J Roentgenol. 1999;172:1365-9.

8. Langerak SE, Vliegen HW, de Roos A, Zwinderman AH, Jukema JW, Kunz P, et al. Detection of vein graft disease using high-resolution magnetic resonance angiography. Circulation. 2002;105:328-33.

9. Debatin JF, Strong JA, Sostman HD, Negro-Vilar R, Paine SS, Douglas $\mathrm{JM}$, et al. MR Characterization of blood flow in native and grafted internal mammary arteries. J Magn Reson Imaging. 1993;3:443-50.

10. Ishida N, Sakuma H, Cruz BP, Shimono T, Tokui T, Yada I, et al. MR flow measurement in the internal mammary artery-to-coronary artery bypass graft: comparison with graft stenosis at radiographic angiography. Radiology. 2001;220:441-7.

11. Langerak SE, Vliegen HW, Jukema JW, Kunz P, Zwinderman AH, Lamb HJ, et al. Value of magnetic resonance imaging for the noninvasive detection of stenosis in coronary artery bypass grafts and recipient coronary arteries. Circulation. 2003;107:1502-8.

12. Vrachliotis TG, Bis KG, Alibadi DA, Shetty AN, Safian R, Simonetti O. Contrast-enhanced breath-hold MR angiography for evaluating patency of coronary artery bypass grafts. Am J Roentgenol. 1997;168:1073-80.

13. Brenner P, Wintersperger B, von Smekal A, Agirov V, Böhm D, Kreuzer E, et al. Detection of coronary artery graft patency by contrast enhanced magnetic resonance angiography. Eur J Cardiothorac Surg. 1999;15:389-93.

14. Rubinstein RI, Askenase AD, Thickman D, Feldman MS, Agarwal JB, Helfant RH. Magnetic resonance imaging to evaluate patency of aortocoronary bypass grafts. Circulation. 1987;76:786-91.

15. Galjee MA, van Rossum AC, Doesburg T, Hofman MBM, Falke THM, Visser CA. Quantification of coronary artery bypass graft flow by magnetic resonance phase velocity mapping. Magn Reson Imaging. 1996;14: 485-93. 
16. Aurigemma GP, Reichek L, Axel L, Schiebler M, Harris C, Kressel HY. Noninvasive determination of coronary artery bypass graft patency by cine magnetic resonance imaging. Circulation. 1989;80:1595-602.

17. Langerak SE, Kunz $P$, Vliegen HW, Lamb HJ, Jukema JW, van der Wall EE, et al. Improved MR flow mapping in coronary artery bypass grafts during adenosine-induced stress. Radiology. 2001; 218:540-7.

18. Takagi T, Yoshikawa J, Yoshida K, Akasaka T. Noninvasive assessment of left internal mammary artery graft patency using duplex Doppler echocardiography from supraclavicular fossa. J Am Coll Cardiol. 1993;22:1647-52.
19. Bach RG, Kern MJ, Donohue TJ, Aguirre FV, Caracciolo EA. Comparison of phasic blood flow velocity characteristics of arterial and venous coronary artery bypass conduits. Circulation. 1993;88(Suppl): II133-40.

20. Crowley JJ, Shapiro LM. Noninvasive assessment of left internal mammary artery graft patency using transthoracic echocardiography. Circulation. 1995;92(Suppl):II25-30.

21. Hoogendoorn LI, Pattynama PMT, Buis B, van der Geest, van der Wall EE, de Roos A. Noninvasive evaluation of aortocoronary bypass grafts with magnetic resonance flow mapping. Am J Cardiol. 1995;75:845-8.

\section{Authoritative}

The Journal of Thoracic and Cardiovascular Surgery is the most frequently cited thoracic/cardiovascular surgery journal in the Science Citation Index. An article in JTCVS is cited on average almost twice as often as those in the closest cardiothoracic journal. 